\title{
Opportunities and Challenges of Functional Foods and Nutraceuticals Development during Covid-19 Pandemic
}

\author{
Lily Arsanti Lestari ${ }^{1,2 *}$ \\ ${ }^{1}$ Department of Nutrition \& Health, Faculty of Medicine, Public Health, \& Nursing, Universitas Gadjah Mada, Jl. Farmako, Sekip Utara, \\ Sleman 55281 \\ ${ }^{2}$ Center of Excellence Institute for Halal Industry \& System, Universitas Gadjah Mada, Gedung LPPT, J1. Kaliurang Km 4, Sleman 55281
}

Functional foods and nutraceuticals demand during the Covid-19 pandemic are increasing tremendously. This is showed that there is a shifting food consumption pattern into healthier food rather than only to satisfy hunger. Functional foods are foods that offer health benefits beyond their nutritional value, whereas nutraceuticals are commodities derived from foods, but are used in the medicinal form of pills, capsules, or liquids that demonstrated physiological benefits. Nutraceutical products consist of isolated bioactive substances such as vitamins, minerals, amino acids, and fatty acids as well as dietary supplements such as probiotics, prebiotics, antioxidants, and enzymes. As the second-largest country with the highest biodiversity, Indonesia has some natural resources that could be developed as functional food or nutraceutical ingredients such as ginger, cinnamon, lemongrass, honey, Moringa oleifera leaves, etc. Indonesian society has indigenous knowledge of the health effect of any plants and spices such as the health effect of "jamu", a functional drink made from several parts of plants. However, the development of functional foods and nutraceuticals in Indonesia has so many challenges concerning regulation. The National Agency for Drug and Food Control of Indonesia did not regulate functional foods and nutraceuticals. However, they have some regulations about processed food with the claim, supplements, natural herbal, and food for medicinal uses. Hence, functional foods and nutraceuticals producers must adjust to the existing regulation. The research and development of functional foods and nutraceuticals consist of several steps namely identification of bioactive compounds, assessment of physiological effect, product formulation and processing, and clinical trial to meet the regulatory demand. The research activities will provide scientific evidence to prove food and nutraceutical functionality. Market demands are also important to obtain an innovative product that meets the consumer's demand. Recent methods to develop functional foods and nutraceutical are "design thinking" methods, a method that focuses problem solving on human rather than on technology or organization. The typical design thinking protocol consists of 3 steps namely observation and synthesis, visualization and rapid prototyping, as well as revising and refining. In the first step, nutraceutical developers must understand the characteristic of targeted consumers. In the second step, models or sketches as well as the early version prototypes need to be created. Gathering feedback on the strengths and weaknesses of the product will lead to the necessary corrections before product commercialization. In the third step, a team from many different backgrounds and specializations can be involved to revise and refine the new product. A Penta helix collaboration between academia, industry, citizen, public authorities, and non-government organization (NGO) can be used as a model of networking in functional foods and nutraceuticals development. The use of "design thinking" methods will minimize the risk of product failure in the market. One of our experiences in functional food development is the exploration of glucomannan from Porang tubers (Amorphophalus oncophylus) as a functional ingredient. Glucomannan, a water-soluble fiber that can be fermented, is extracted from Konjac (Amorphophallus konjac) tuber or root. However, in Indonesia glucomannan can be extracted from Porang tuber that can be found in many areas. In the beginning, we collaborate with the local farmer, NGO, and governments to optimize the production of Porang tubers. After we found the method to extract the glucomannan, we develop several food products namely jelly, noodle, rice analog, etc and analyze the physical, chemical, microbiological, and sensory characteristics. The jelly product containing glucomannan was tested for the clinical trial. We found that this jelly could lower the body weight and body mass index of the overweight and obese subjects as well as maintain the level of blood glucose, the total cholesterol, LDL cholesterol, and triglycerides. The health benefit of this jelly can be used as the rationale for health claimed. It is important to collaborate with the food industry to commercialize this product. In conclusion, Indonesia needs to develop guidelines for Functional Food and Nutraceuticals Development, this guideline can be used as a reference for producers for claims on nutrition and health. For researchers, academia, and the food industry that

\footnotetext{
*Corresponding email: lily_al@ugm.ac.id
} 
will develop a new functional food and nutraceutical product, it is important to plan and know the targeted consumer so that the new product will succeed in the market. 